\title{
UN INTEGRAFO.
}

\section{PIO SCATIZZI.}

Fu in occasione delle equazioni integrali del Volterra che nacque il bisogno di costruire apparecchi i quali eseguissero l' integrale del prodotto di due funzioni e, dato il fenomeno dell' eredità, riuscissero ad ottenere, almeno per pnnti la curva integrale. Non si può infatti operare l'inversione dell' equazione

$$
\varphi(x)=u(x)+\int_{0}^{x} \mathrm{~K}(x-\xi) u(\xi) d \xi
$$

e quindi costrairsi il nucleo risolvente

$$
\mathrm{S}(t-x)=\sum_{i=1}^{\infty} \mathrm{K}^{(i)}(x-\xi)
$$

senza eseguire l' integrale

$$
k^{(i)}(v)=\int_{0}^{v} k^{(i)}(v-u) \cdot k^{(i-j)}(u) d u
$$

in cui

$$
z-\xi=u \quad x-\xi=v
$$

onde poi si possa ottenere la

$$
u(x)=\varphi(x)+\int_{0}^{x} \mathbf{S}(x-\xi) \varphi(\xi) d \xi .
$$

Il Prof. Pascal in una sua recente ed originale pubblicazione ') descrive un apparecchio che soddisfa al quesito.

1) Pascal. "I miei integrafi ". Tipografia Pellerano, Napoli 1914. 
$\mathrm{Mi}$ è sembrato ancora di qualche interesse proporre un meccanismo che risolva il problema nella sua generalità, senza sagome ricurve, solo a mezzo di carrelli e righe rettilinee.

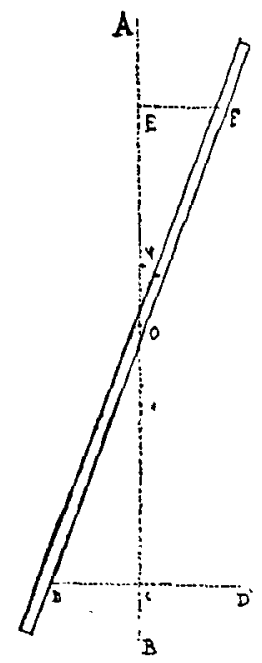

Fig. 1.

Il principio su cui si fonda $\dot{\theta}$ il seguente: Sia una verghetta $D F$ girevole intorno ad un punto fisso $O$. Sia $p$ l'angolo azimutale variabile che può divenire negativo dall'altra parte della A B. Scegliamo sulla A B un altro punto fisso $G$, e sia $\mathrm{OC}=a$ costante. Se ora facendo girare la verghetta intorno ad $O$, la normale $D C$ ad $O C$ varia secondo una funzione data $\chi(x)$ sarà sempre

$$
\chi(x)=a \operatorname{tag} \varphi .
$$

Inoltre se la distanza OE si fa variare proporzionalmente ad una seconda funzione $\psi(x)$, la perpendicolare E F ad $A O$ verificherà alla

$$
\mathrm{EF}=\psi(x) \operatorname{tag} \varphi
$$


sostituendo il valore di $\operatorname{tang} \varphi$ ricavato dalla (1) otterremo

$$
\mathrm{EF}=\frac{1}{a} \chi(x) \psi(x) \text {. }
$$

Per valori negativi delle funzioni basterà fare scorrere $\mathbf{i}$ punti rispettivamente sul prolungamento di C D ossia lungo $\mathrm{CD}$ e sopra $\mathrm{OB}$ prolungamento di OA. Infatti per ciascuno dei detti spostamenti negativi si ottiene pure un oscillazione negativa del prodotto, si effettua cioè nel semipiano sinistro di A B.

Si vede poi che EF si annulla coll' annullarsi di una delle due funzioni; infatti per la (1) $\dot{e} \chi(x)=0$ per $\psi=0$ ed allora anche $\mathrm{EF}=\mathrm{O}$. Lo stesso si ha per $\mathrm{OE}=\mathrm{O}$.

L'integrafo pertanto può costruirsi nel seguente modo: (vedi fig. 2).

Sopra il lato A B di un rettangolo di metallo mobile su due ruote il cui asse è parallelo a questo lato, scorrono due indici che seguono le due curve $\chi(x)$ e $\psi(x)$, mediante due verghette $L L^{\prime}$ collegate rigidamente a due carrelli scorrenti su A B. Sul lato C D si muove il carrello integrale T. In O, punto fisso rigidamente al rettangolo metallico è girevole radialmente una doppia squadra $h h \mathrm{OL}$ comandata per la forcella in L dall'indice che segue la $\chi(x)$ e che rimane sempre a distanza fissa dal lato A B. Sicchè se l'asse $x$ della medesima curva è normale a questo lato, le ordinate della $\chi(x)$ sono proporzionali alla tangente dell' angolo formata da $h h$ ed A B. Normalmente alla verga E L' dell' altro carrello di $\psi(x)$, viene fissata un' altra doppia squadra F G K E scanalata lungo la traversa $F G$. In tale scanalatura scorre una punta $\mathrm{V}$ fissa ad un carrello che può muoversi lungo la verga $h h$. Nel medesimo punto $V$ si appoggia un' altra squadra VH M che dà poi la direzione mediante parallelogrammo al piano della rotella integratrice in $\mathrm{T}$. Tale squadra può rotare intorno al perno fisso $\mathrm{H}$. Bisogna notare che il punto $\mathrm{Q}$ giace sul prolungamento $\mathrm{EK}$, per $\psi=0$ il punto $\mathrm{K}$ cade in $\mathrm{O}$ quindi la distanza $\mathrm{OK} \mathrm{mi}$ da la $\psi(x)$. Dunque $\mathrm{KV}$ è il prodotto $\psi \chi$. Il triangolo $\mathrm{VKH}$ ̀े il triangolo di Abaka- 
nowicz in cui $\mathrm{KH}$ è fissa e rappresenta la costante di integrazione, KV l' ordinata da integrarsi, basterà dunque che

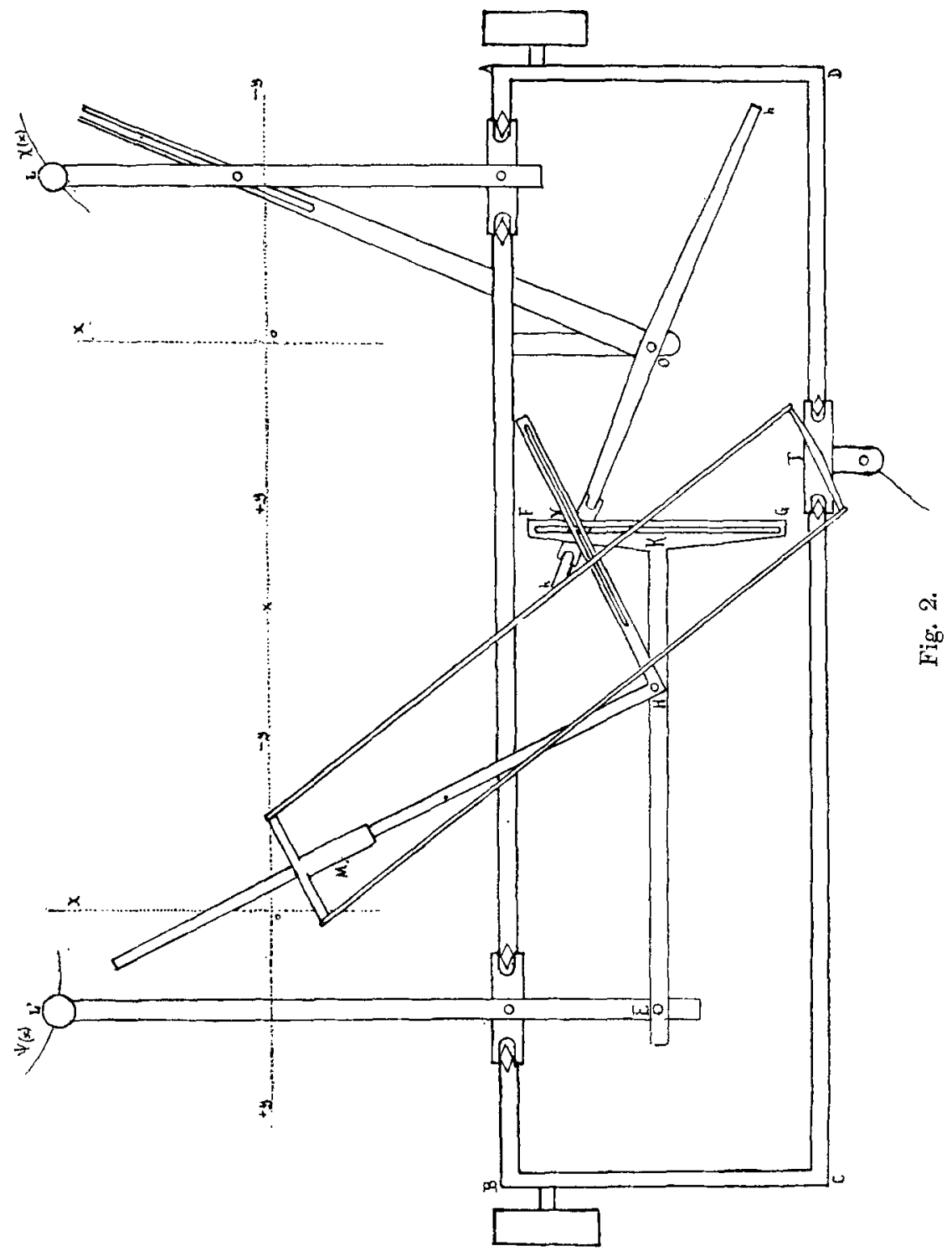

iI piano della rotella si mantenga parallelo all'ipotenusa $\mathrm{VH}$. 
Nel nostro caso però siccome la base costante $\mathrm{KH}$ non giace lungo la direzione dell'asse $x$ ma è ad essa normale si dovrà rendere parallelo il piano della rotella all' altro cateto della squadra HM.

Ora se si dà al piano della rotella integratrice un angolo $\alpha=\operatorname{arctg} m$ colla ipotenusa del triangolo fondamentale nel solito integrafo di Abakanowicz si viene ad integrare ') l' espressione

$$
y=\int \frac{a m+\mathrm{Q}(x)}{a-m \mathrm{Q}(x)} d x+\mathrm{C} \text { ost. }
$$

Nel nostro caso essendo $Q(x)=\psi(x) \chi(x)$ verremo ad integrare l' equazione :

$$
y=\int \frac{a m+\psi(x) \chi(x)}{a-m \psi(x) \chi(x)} d x .
$$

Se ora nella (3) dividiamo tutto per $m$ il secondo membro e andiamo al limite $m=\infty$ avremo $\alpha=90^{\circ}$ e si otterrà

$$
y=-a \int \frac{d x}{\mathrm{Q}(x)}
$$

ossia per il nostro apparecchio

$$
y=-a \int \frac{d x}{\psi(x)} \frac{x(x)}{\chi(}
$$

Facendo ritornare $\alpha=0$ e posto $\psi(x)=x$ e $\chi(x)=3 x$ si ha l' equazione

$$
\frac{d y}{d x}=3 x^{2} \quad \text { integrando } \quad y=x^{3} .
$$

Può quindi l' istrumento servire per compasso parabolico cubico.

Facciamo ora una prima modificazione nell' apparecchio.

1) V. « Integrafi a perno fisso » nell' opera citata $§ 11$. 
Asportiamo la doppia squadra EKGF ed intorno ad un perno in $\mathrm{E}$ sia girevole una squadra col lato $\mathrm{EV}$ (fig. 3)

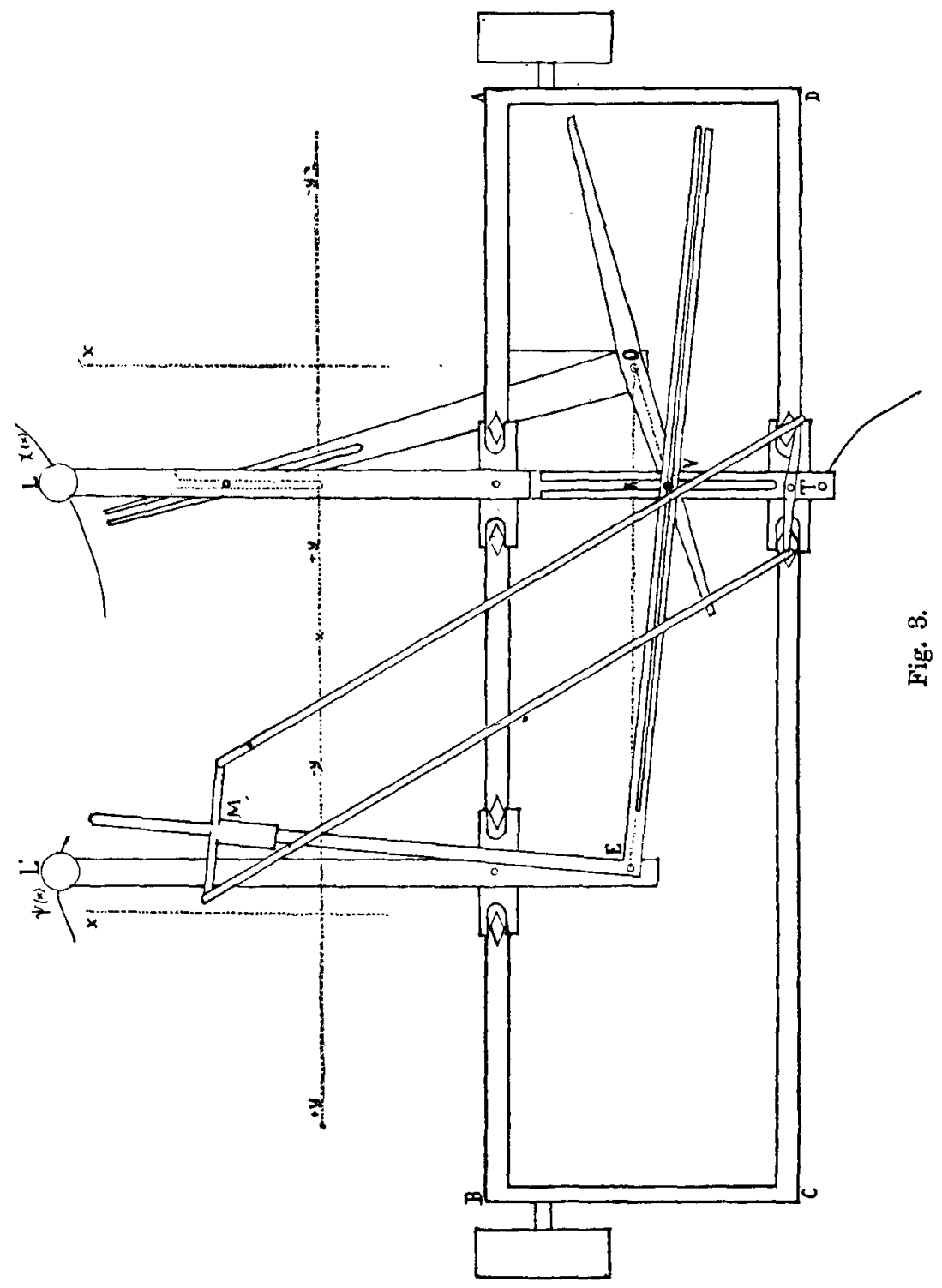

prolungata convenientemente (due volte circa la $h h$ ) in modo 
che possa essere sempre comandato dal perno in V. Fissiamó poi sul carrello integrale una riga rettilinea e tagliata a forcella in modo che comandi il perno in $\nabla$. Il triangolo che ne risulta $\mathrm{E} \mathrm{K} \mathrm{V} \mathrm{ci} \mathrm{dà} \mathrm{un'} \mathrm{equazione}$

$$
\frac{d y}{d x}=\frac{\chi(x) y}{n+y+\psi(x)}
$$

Lo zero della curva integrale deve cadere sul piede della perpendicolarc abbassata da $\mathrm{O}$ sul lato $\mathrm{CD}$, ed il carrello integrale non deve oscillare più della lunghezza $h h$. La costante $n$ rappresenta la distanza dei due assi $x$ delle due curve $\chi(x)$ e $\psi(x)$. Il numeratore del secondo membro dipende dal prodotto di $\chi(x)$ con $\mathrm{KO}=y$. Il deneminatore che rappresenta la lunghezza $\mathrm{E} \mathrm{K}$ è uguale alla distanza $n$ più la somma algebrica di $y$ e di $\psi(x)$.

Dando alla rotellina integratrice un angolo coll'ipotenusa $\alpha=\operatorname{artag} m$ abbiamo l'altra equazione

$$
\frac{d y}{d x}=\frac{m n+m[y+\psi(x)]+\chi(x) y}{n+y+\psi(x)-m \chi(x) y}
$$

per $m=\infty$ avremo:

$$
\frac{d y}{d x}=-\frac{y+\psi(x)+n}{\chi(x) y}
$$

Sembra bene di far osservare che l'uso delle sagome ricurve le quali servono a dare la direzione al piano della rotellina integratrice, condurrà spesso a soluzioni brillanti e generali dal lato analitico come si può vedere in una recente nota del Dott. Aiello ') per le equazioni di Riccati e di Abel ed in generale quelle che si possono mettere sotto la forma

$$
\eta^{\prime}=(Q(x)-y)^{n}
$$

1) C. Aiello. "Su di una importante applicazione dell' integrafo Pascal a riga curvilinea ». (Rend. della R. Acc. di Napoli), (3), v. 18, 1912. 
Ma quanto poi alla pratica, a cui è rivolto lo studio degli integrafi, i risultati sono forse più limitati di quello che si crede. Come nota il Pascal si possono dare delle sagome di curve che hanno bisogno di un manubrio aggiunto e collocato opportunamente per ovviare a scorrimenti automaticamente impossibili. Vi $\dot{\theta}$ anche qualche difficoltà di più, perchè può darsi, come per esempio per la curva dell'equazione di Abel ed altre simili, il caso in cui la tangente di questa in qualche piccolo tratto si conservi quasi perpendicolare al raggio vettore per modo che con tutto il manubrio non si sappia se si debba far ruotare la sagoma in un senso ovvero nel senso opposto. Così anche può darsi il caso che si trovino dei punti morti ossia che il carrello differenziale venga impedito di scorrere mentre segue la curva del disegno $\varphi(x)$. Basterà infatti che le tangenti $t^{\prime}$ della curva integrale e $t$

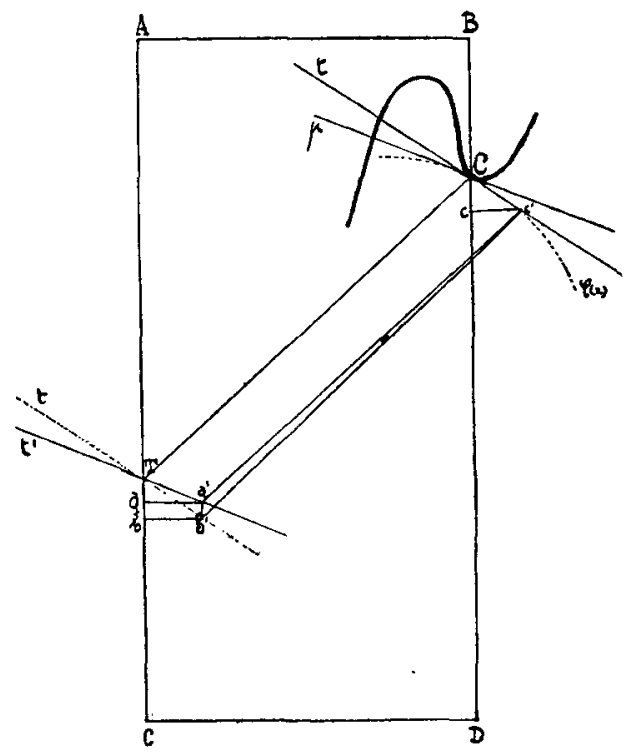

Fig. 4.

della $\varphi(x)$ in $\mathrm{C}$ divengano convergenti, (vedi fig. 4). Se chiamiamo $\mathrm{T}$ il lapis del carrello integrale, $\mathrm{C}$ l' indice del differenziale $\theta$ la curva descritta in figura sia la sagoma metallica 
rigidamente connessa col raggio vettore $\mathrm{TC}$, (se la sagoma si esprime in coordinate polari, $\mathrm{T}$ ne $\dot{e}$ il polo): allora per un piccolo spostamento dell'apparecchio normale a B D uguale a $\mathrm{O}^{\prime}=a a^{\prime}=b b^{\prime}$ occorre portare la punta $\mathrm{C}$ in $c^{\prime}$ e quindi scendere sulla guida $\mathrm{BD}$ fino a $c$. $\mathrm{Ma}$ il punto $\mathrm{T}$ del carrello integrale invece di venire in $b^{\prime}$ e scendere di $\mathrm{T} b=\mathrm{C} c$ giunge solo in $a^{\prime}$ scendendo di $\mathrm{T} a$, ed allora essendo le due $t$ in $\mathrm{C}$ e in $\mathrm{T}$ parallele, sara $b^{\prime} c^{\prime}=\mathrm{T} \mathrm{C}$ e $a^{\prime} c^{\prime}<b^{\prime} c$ come si vede dal triangolo $b^{\prime} a^{\prime} c^{\prime}$ che sarà in generale scaleno. Ora se in $\mathrm{C}$ la sagoma sta vicino ad un punto di flesso in modo che la curva rimanga tutta dalla parte superiore, allora comunque si faccia ruotare col manubrio la sagoma, il raggio vettore non potrà mai diminuire come si richiederebbe nel nostro caso. La difficoltà opposta si riscontrerebbe al di là del punto di flesso nel caso che la derivata di $\varphi(x)$ in $c$ fosse crescente, perchè allora la sagoma tenderebbe invece a far accorciare il raggio vettore come è chiaro.

La sagoma data dal Pascal rappresenta una curva del terzo ordine, in cui i punti di flesso sono dati dalla formola

essendo

$$
u=\frac{\mathrm{P}+m \omega+n \omega^{\prime}}{3}
$$

$$
u=\int_{x_{0} y_{0}}^{x y} \frac{d x}{\bar{F}_{y}^{\prime}}
$$

un integrale Abeliano di prima specie, $\mathrm{F}(x y)$ la cubica in questione (quindi senza singolarità); $\mathrm{P}=$ cost ; $\omega^{\prime}$ e $\omega$ periodi ciclici $; m$ ed $n$ due numeri interi che non possono prendere altri valori che $0,1,2$. Facendo tutte le combinazioni possibili di questi valori, abbiamo nove valori distinti di $u$ e quindi nove punti di flesso. Ma per ogni punto di flesso vi sono due possibilità d' inconvenienti, in tutto 18 archetti in cui si verifica la poca attuabilità dell' apparecchio '). Biso-

') L' unico caso in cui data la invariabilità del raggio vettore l' inconveniente suddetto non si verificherebbe è dato quando le derivate di $\varphi$ e dell' integrale hanno lo stesso valore. 
gnerebbe dunque dimostrare che esiste almeno una posizione della sagoma in cui questi inconvenienti sono eliminati.

L' equazione (8) dà il modo di integrare l'equazione di Abel conosciuto però un integrale particolare.

Se nell' equazione di Abel

$$
y^{\prime}+\mathrm{A} y^{3}+\mathrm{B} y^{2}+\mathrm{C} y+\mathrm{D}=\mathrm{O}
$$

in cui A B C sono funzioni della sola $x$, si fa $y=u+v$, avremo :

$$
\begin{aligned}
& u^{\prime}+v^{\prime}+\mathrm{A}\left(u^{3}+3 u^{2} v+3 u v^{3}+v^{3}\right)+ \\
& +\mathrm{B}\left(u^{2}+2 u v+v^{2}\right)+\mathrm{C}(u+v)+\mathrm{D}=\mathrm{O}
\end{aligned}
$$

se $u$ è un integral particolare l' equazione

$$
u^{\prime}+\mathrm{A} u^{3}+\mathrm{B} u^{2}+\mathrm{C} u+\mathrm{D}=\mathrm{O}
$$

sarà identicamente soddisfatta rimarrà allora:

$$
v^{\prime}+\mathrm{A}\left[v^{3}+(3 \mathrm{~A} u+\mathrm{B})\right] v^{2}+\left(3 u^{2} \mathrm{~A}+2 u \mathrm{~B}+\mathrm{C}\right) v=0
$$

chiamando

$$
\mathrm{A}=\alpha, \quad 3 \mathrm{~A} u+\mathrm{B}=\beta, \quad 3 u^{2} \mathrm{~A}+2 u \mathrm{~B}+\mathrm{C}=\gamma
$$

e dividendo tutto per $v$ avremo l' equazione

$$
\frac{d \log v}{d x}+\alpha v^{2}+\rho v+\gamma=0
$$

per $v=e^{\eta} \quad$ sarà $\eta^{\prime}+\alpha e^{2 \eta}+\beta e^{n}+\gamma=0$. Facendo

$$
\eta=\xi-\int \gamma d x
$$

e sostituendo avremo :

$$
\xi^{\prime}-\gamma+\alpha e^{-2 \int \gamma d x} e^{2 \xi}+\beta e^{-\int \gamma d x} e^{\xi}+\gamma=0
$$


ponendo

$$
\Phi_{1}=\alpha e^{-2 \int \gamma d x} \quad \Phi_{3}=\rho e^{-\int \gamma d x}
$$

resterà

$$
\xi^{\prime}+\Phi_{1} \mathrm{C}^{2 \xi}+\Phi_{2} e^{\xi}=0
$$

moltiplicando tutto per $e^{\xi}$ e ponendo $e^{\xi}=\varphi$ sarà

$$
\begin{gathered}
\varphi^{\prime}+\Phi_{1} \psi^{3}+\Phi_{2} \varphi^{2}=0 \text { poncudo } \frac{1}{\varphi}=\theta \text { nella } \\
\frac{1}{\varphi}\left(\frac{d}{d x}\left(\frac{1}{\varphi}\right)\right)+\Phi_{2} \frac{1}{\varphi}+\Phi_{1}=0
\end{gathered}
$$

avremo

$$
\theta \theta^{\prime}=\Phi_{2} \theta+\Phi_{1} \quad \text { ossia } \quad \theta^{\prime}=\frac{\Phi_{1}}{\theta}+\Phi_{2} \quad(\mathrm{~K}) .
$$

Di questa equazione non si può dare che un fattore integrante :

$$
\mu=\frac{\theta}{[\theta+f(x)]^{n}} \quad \text { in cui } f(x)=-n \frac{\Phi_{1}}{\Phi_{2}}, n=\text { cost }
$$

quando

$$
\Phi_{2}=\frac{-n^{2}}{1-n^{2}} \frac{d}{d x}\left(\frac{\Phi_{1}}{\Phi_{2}}\right)
$$

Negli altri casi ci si può servire dell' integrafo mediante il dispositivo espresso dall' equazione (8). Infatti questa è simile alla $(\mathrm{K})$ ciò̀ si può disporre:

$$
\frac{d y}{d x}=\frac{-(n+\psi(x))}{\chi(x)} \frac{1}{y}+\frac{-1}{\chi(x)}
$$

basterà fare

$$
\Phi_{1}=\frac{-(\psi(x)+n)}{\chi(x)}=\frac{\alpha}{e^{2 \int \gamma d x}} \quad \Phi_{2}=\frac{-1}{\chi(x)}=\frac{\beta}{e^{\int \gamma d x}}
$$


sarà allora

$$
\Psi(x)=\frac{\alpha}{\beta} e^{-\int \gamma d x} \quad \chi(x)=\frac{-1}{\beta} e^{\int \gamma d x} .
$$

Se in questo medesimo dispositivo si allunga la riga del carrello integrale finchè vada per mezzo di forcella a catturare l' indice della $\chi(x)$, come si vede nella fig. 2 dove si rappresenta il prolungamento con linee punteggiate, si ottiene, facendo $\chi(x)=y$ l' equazione:

$$
\frac{d y}{d x}=\frac{y^{2}}{y+\psi(x)+n}
$$

dando al piano della rotellina un angolo $\alpha=\operatorname{artag} m$ otterremo :

$$
\frac{d y}{d x}=\frac{m[y+\psi(x)+n]+y^{2}}{n+\psi(x)+y-m y^{2}}
$$

per $m=\infty$ si ha:

$$
\frac{d y}{d x}=-\frac{n+\psi(x)+y}{y^{2}} .
$$

